\title{
Standards for the Design of Tracking, Telemetry and Command Subsystem of Spacecraft
}

\author{
A. V. Mishurov, S. P. Panko, A. V. Khnykin \\ Siberian Federal University \\ Krasnoyarsk, Russia \\ AMishurov@sfu-kras.ru
}

\begin{abstract}
A lag of CCSDS recommendation is shown. Main part of onboard equipment - Tracking, Telemetry and Command Subsystem is shown as source for potential improvements for new standards. It is possible by increasing of estimation accuracy of the parameters of the orbit of spacecraft.
\end{abstract}

Keywords-spacecraft; Ground Control; telemetry; range tracking; CCSDS.

\section{INTRODUCTION}

Spacecrafts, as complex high-tech products, require a systematic approach to design and production. In the current context, each spacecraft has unique operational characteristics inherent only to it. System theory and system engineering are the basic approaches that should control the whole process: from the choice of the generalized model, to the carry out of the machine for finishing tests and quality control. Such systems are costly and their serial production is not advisable for financial reasons or the narrowness of the field of application. An attempt to take into account both the requirements of the customer and the requirements of the regulatory documents lead to contradictions in the choice of specific implementation technologies. Therefore, the management of requirements in accordance with GOSTs, technical conditions, working documentation and other normative documents for limited or piece production of modern equipment is a real problem.

What is the problem with managing the standard, if it guarantees quality? It is known that any developing an open system, which requires management needs to develop adequate operational content feedbacks. Since science and technology is not standing still, and the decisions that were best yesterday, the next day often become a losing one. In the conditions of market competition, this is a serious factor affecting the consumer properties of products. Therefore, standards should be revised. But how fast and often? Obviously, if the normative documents will rewrite with the appearance of each new solution, then the probability of error becomes high: insufficiently developed technological processes do not always immediately reveal the drawbacks of innovations. On the other hand, revision of normative documents can last for years and decades, and new opportunities cannot be used only because of current restrictions (especially in the field of information technology).

Many states have developed and successfully use their own standards of satellite technology to coordinate the activities of operating organizations and industrial enterprises, producing different components of spacecraft. However, as satellite technology is the common achievement of mankind, there was a necessity for international cooperation of participants of the national space programs. As result was the foundation in 1982 of The Consultative Committee for Space Data Systems (CCSDS), the general purpose of which is to coordinate activities in this area [1]. The CCSDS is composed of 11 national space agencies, 28 observer-states and more than 140 industrial partners now. CCSDS is an international forum for the development of standards for communications, data transmission and space flight.

The CCSDS standards library, published in Russianlanguage transcription, contains the following sections [8]:

1. CCSDS 320.0-B-5. Global Spacecraft Identification Field ("Pink Book")

2. CCSDS 211.1-B-3. Proximity-1 Space Link Protocol

3. CCSDS 414.1-B-1. Pseudo-Noise (PN) Ranging Systems

4. CCSDS 414.0-G-0. Pseudo-Noise (PN) Ranging Systems

5. CCSDS 301.0-B-4. Time Code Formats.

From these sections, the sections dealing with the space link protocols and the sections on range-measurement can be most flexibly developed. The space link protocols should take into account the real velocity of command and telemetry transmission, which will ensure a uniform loading of the transmitter-receiver and create the prerequisites for increasing the efficiency of measuring current navigation parameters. Procedures for range-measurements can be optimized by the types of signals and methods of their processing. The objective point of these tasks should be to ensure a high accuracy in measuring the range and velocity of a spacecraft in real time.

Sections 1 and 5 of CCSDS standards libraries are less likely to be modernized, as they affect global interests, but this possibility should not be neglected as necessary.

Development, manufacture and operation of the spacecraft, located at a considerable distance from the Ground Control and working in the aggressive environment of high vacuum forming a special requirements compared to ground equipment available to renovation and to control the availability. At current time matured sufficiently large active lifetime up to 15 years, achieved is not only using special strength construction materials or highly reliable of microelectronic products, and redundancy components and subsystems too. In addition, an 
important role has the operation of work systems and spacecraft components, providing a change of operating modes if it is caused by the necessity to restore at least some of the functions. For implementation these requirements, parameters and modes of the spacecraft continuously transmitted to the Ground Control, where they are controlled by operating personnel. If necessary, adequate measures is takes by sending appropriate commands to the spacecraft. These functions are performed by TT\&C - tracking, telemetry and command subsystem.

\section{TRACKING, TELEMETRY AND COMMAND SUBSYSTEM}

TT\&C transmits commands to the spacecraft by base on dedicated radio channel. Onboard part of TT\&C provides a transmission the telemetry data from the sensors and subsystems of spacecraft and payload to Ground Control. In addition, the TT\&C of the spacecraft is charged the measuring the current navigational parameters of the spacecraft, primarily the range and velocity. Operation of onboard part of TT\&C is supported by another independent radio channel.

Control of operating modes and functions of the spacecraft is carried out by transferring commands and flight tasks from the Ground Control to the spacecraft in accordance with the generalized structural diagram of the onboard part of the TT\&C (Fig. 1), developed in accordance with the recommendations of CCSDS [1].

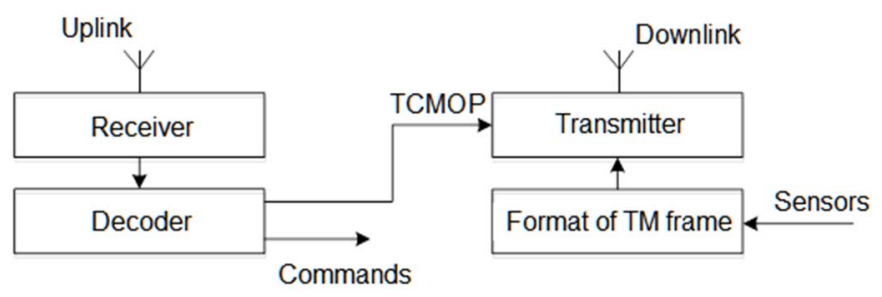

Fig. 1. Generalized block diagram

Functionally, TT\&C consists of two segments: Onboard equipment and segment of Ground Control. Commands and the initial range-measurement signal are transmitted on the same carrier frequency via the Uplink on the spacecraft. The received signals are decrypted, and the allocated commands are sent for execution by the nodes and subsystems of the spacecraft. The allocated range-measurement signal on the internal line of the TCMOP (Transmission Channel for Measuring the Orbital Parameters) is emitted from the Transmitter via the Down link to the Ground Control.

Telemetry subsystem is responsible for data collection from a wide variety of sensors which extract information about the pressure in the fuel tanks, voltage and consumed currents of transmitters, radiating power of the transmitter, the critical voltages and currents, the temperature in the different subsystems, the status of switches and movers, sensors of lifesupport systems, sensors of other engineering subsystems on board spacecraft. The birth of microprocessor-based equipment led to productivity gain of data handling, as a result was increased independence of the spacecraft and the complexity of the spacecraft's mission. The position of the spacecraft and its evolution are also monitoring by telemetry.

The command channel of the spacecraft works as follows. The signal from the receiving antenna is received at the input of the receiver of spacecraft; the signal is demodulated and decoded. In Decoder, the reliability of the decoded commands is evaluated and the commands are distributed according to their intended purpose.

On the response radio channel, which is commonly referred to as telemetry, receipts on the reception and execution of commands, reports on the operating modes, the functions to be performed and telemetric information about the current parameters of the onboard nodes and systems are transmitted to the Ground Control. Onboard equipment of TT\&C supports the measurement of orbit parameters - the current navigational parameters of spacecraft. The range of spacecraft is measured on the basis of an estimate of the time delay for passing a test signal along the Ground Control - Spacecraft - Ground Control route, minus the delay in the Onboard equipment. The velocity of the spacecraft, as well as the range of spacecraft, are measured in the Ground Control on the basis of the processing of signals coming from the spacecraft.

CCSDS main position on the tracking system, telemetry and command transmission from the Ground Control to the spacecraft concentrated in providing greater automation within the individual spacecraft relative to earlier decisions not only, as well as to ensure the unity of the information base of different subsystems, which leads to greater cross-support facilities and services.

By recommendations of CCSDS, in accordance with the OSI seven-layer model [2], telemetry system of spacecraft is based on two main conceptual categories: "packet telemetry" and "telemetry channel coding."

Packet telemetry provides efficient data transmission received from sources in a standardized form and is characterized by a high degree of automation by using of protective coding and detecting and correcting errors that occurred during propagation. Packet telemetry provides a mechanism for the implementation of common data structures and protocols that can contribute to the development and operation of TT\&C [3]. Telemetry Channel Coding provides the processing of raw data so that individual messages are easily distinguished from each other. This makes it possible to reconstruct the data transmitted from the spacecraft to Ground Control with a low error probability, thereby improving channel performance. In addition, the channel coding is aimed at protecting the telemetry channel from possible tampering.

\section{ESTIMATION OF THE PARAMETERS OF THE ORBIT}

Estimation of the parameters of the orbit of spacecraft is based on measurements of the range tracking and the radial component of its velocity of spacecraft. To measure the range and velocity of the spacecraft relative to Ground Control, a

The publication is supported by Krasnoyarsk Region Science and Technology Support Fund. 
TCMOP is provided between the receiver and transmitter nodes.

There are various approaches to the implementation of Onboard equipment of TT\&C are known. In [4], in particular, the command signal and the audio signal, which can be considered as a range-measurement signal, are summed and transmitted at a common subcarrier frequency. Otherwise, the Ground Station and spacecrafts paths are little different from the usual superheterodyne receiver. In another case, broadband signals are used to transmit the command and rangemeasurement signals on one common carrier. The command signal is modulated by the first broadband code, and the rangemeasurement signal by the second; the length of the second code is a multiple of the length of the first code. The carrier modulation comprises modulating one or more subcarriers with a plurality of range-measurement tones. Signals with BPSK / FM modulation occupy a sufficiently wide frequency band, about $800 \mathrm{kHz}$ for a FM with a deviation of $\pm 400 \mathrm{kHz}$, which requires a bandwidth of up to $15 \mathrm{MHz}$ to control a constellation of 11 spacecrafts. Broadband signals reduce the acuteness of the problem. In [6], serial transmission of signals is used, to which both command and range-measurement signals can be referred.

In general, technical solutions for TT\&C of spacecraft do not relate to system issues, but relate to the measurement, primarily the range. The most common are two approaches using pseudo-random or tone signals for these purposes. A well-known solution for measuring range consists in the emission of several sinusoidal tones by the Ground Station. These tones are demodulated on board and used to remodulate the carrier of telemetry signal on the channel down simultaneously with the telemetry subcarrier. The range is estimated from the measured phase shift at the higher frequency, the so-called "major" tone with refinement in "minor" tones, which correspond with the major tone in a geometric progression of $1 / 5$ on the recommendation of the ESA. The task of removing ambiguity is inherent not only in tone, but also in pseudo-random signals. Ambiguity is that the highest accuracy is obtained at high frequency components. But there is a plurality of readouts. The simplest and most effective procedure for resolving ambiguity by scanning the signal components, starting with the highest frequency.

Range tracking is based on measuring the propagation time of the ranging signal of pseudo-random sequence type along the route Ground Control - Spacecraft - Ground Control [5]. Second variant recommended making the range tracking by estimating the delay of phase of tone signal, radiating and receiving by Ground Control.

It is well known [5] that the accuracy of range tracking is determined by the signal to noise ratio at the correlator output:

$$
q=\frac{E}{N_{0}}
$$

where $N_{0}$ is the power spectral density of the noise source, $N_{0}=$ $2 D_{ш} / \Delta F, D_{ш}$ - noise variance, $2 \Delta F$ - bandwidth occupied by a signal $(-\Delta F \ldots+\Delta F), E-$ energy of signal $s(t)$. In the case of pseudo-random sequence composed of $\mathrm{M}$ chips

$$
E=\int_{0}^{M T} s^{2}(t) d t
$$

where $T$ - the duration of the chip. This implies that improving the accuracy of range tracking according to the CCSDS recommendations provided by increasing $\mathrm{M}$, which is especially important when working with the spacecraft in High Elliptical Orbit. However, the CCSDS recommendations not emphasized the important question of simultaneous transmission of commands and range-measurement pseudorandom sequence, except the using for this purpose a variety of subcarrier frequencies. But this does not meet the requirement of reducing the bandwidth of frequencies. In [6] proposed a solution to this problem by alternately transmitting commands and ranging signal on the same carrier frequency. The disadvantage of this solution is to peak limit the accuracy of range tracking, which is especially evident at the stage of the spacecraft launch to the operating point when control commands are sent to the most intense. At the stage of steady functioning at the operating point is the most productive to use of command and range-measurement pseudo-random sequence of the same duration. It is useful to radiate successively the command and range-measurement sequences and measure the range not only by the range-measurement, but also by command sequences. The highest accuracy is achieved when the lengths of the range-measurement and command sequences are equal.

The second aspect is not dealt with CCSDS, is the accuracy of measuring of radial component of the velocity of the spacecraft, which is determined solely on the basis of measuring the Doppler frequency shift. Studies show that there is room for improving the accuracy of Doppler measurements [7]. In addition, in the process of resolving the ambiguity there is a reserve of increasing the accuracy of Doppler measurements and, accordingly, the velocity of the spacecraft. There have been other inconsistencies to modern level of development of science and technology.

\section{CONCLUSION}

The reason for the lag CCSDS recommendations to new results of intellectual activity lies in a large period since its adoption in 2010. Therefore, there is need to develop a national standard - equivalent of CCSDS, which should take into account the modern achievements of the system capabilities of the development of TT\&C of the spacecraft, as well as give greater freedom to creativity for researchers in order to improve the accuracy of measuring telemetry parameters and current navigation parameters.

At the moment in Russia there GOST 56096-2014 "Space data and information transfer systems", which ensures the harmonization of Russian control systems with the recommendations of CCSDS in the formation and transmission of telemetric information.

This standard establishes rules for the construction and organization of measuring systems on the objects and products of rocket and space equipment and ground space infrastructure 
only in the part of the organization of packet data transmission. However, the standard does not provide sections that recommend the use of modern modulation / demodulation methods that ensure efficient use of the frequency resource, as well as recommendations for improving the accuracy of measuring current navigation parameters.

This caused the expediency of developing its own Russian standard, which is a subspecies of CCSDS. The Russian standard on space systems for data transmission in the part of TT\&C of the spacecraft - the analogue of CCSDS - should take into account the modern achievements of science and technology and contain at least the following sections:

- organization of TT\&C of the spacecraft with the purpose of transfer of command and command-flight information from the Ground Control to the spacecraft;

- modern methods of modulation / demodulation, providing an increase in the efficiency of information transfer with a decrease in the bandwidth of occupied frequencies;

- increasing the accuracy of measuring current navigation parameters, namely, the range and velocity of spacecraft relative to the Ground Control, which provides high accuracy in determining the orbital parameters of spacecraft;
- $\quad$ protection of the command channel from unauthorized interference while maintaining energy performance.

Obviously that the Russian standard should be consistent with the current and newly introduced provisions of CCSDS.

The reported study was funded by Krasnoyarsk Region Science and Technology Support Fund according to the research project: «Preparation for the introduction of the software and hardware complex for automation of tests of the on-board equipment of the tracking, telemetry and command subsystem in JSC "ISS"

\section{REFERENCES}

[1] CSDS. Recommendation for space. Data system standard. A blue book, 2000. A green book, The CCSDS website, www.ccsds.org.

[2] International Standards Organization. www.iso.org.

[3] B. Sklar. Digital communication. Moscow - St. Petersburg - Kiev, 2003. (In Russ.)

[4] T.T. Pham, DSN Chief System Engineer. 203, Rev. C Sequential Ranging DSN Telecommunications Link Design Handbook October 31, 2009.

[5] V.I. Tikhonov. Statistical radios. M. : 1966. (In Russ.)

[6] Harles et al. Ranging system and method for satellites. US Patent 6864838, March, 8, 2005.

[7] S.P. Panko, M.S. Tsimbal. Measurement of the velocity of the spacecraft // The Research of the Science City. 2015. № 4. C. 25-29. 